\title{
A note on a Ramsey-type problem for sequences
}

\author{
Andrzej Dudek* \\ Department of Mathematics \\ Western Michigan University \\ Kalamazoo, MI, USA \\ andrzej.dudek@wmich.edu
}

Submitted: Mar 24, 2014; Accepted: Sep 9, 2014; Published: Sep 18, 2014

Mathematics Subject Classifications: 05A05, 05D10

\begin{abstract}
Two sequences $\left\{x_{i}\right\}_{i=1}^{t}$ and $\left\{y_{i}\right\}_{i=1}^{t}$ of distinct integers are similar if their entries are order-isomorphic. Let $f(r, X)$ be the length of the shortest sequence $Y$ such that any $r$-coloring of the entries of $Y$ yields a monochromatic subsequence that is also similar to $X$. In this note we show that for any fixed non-monotone sequence $X$, $f(r, X)=\Theta\left(r^{2}\right)$, otherwise, for a monotone $X, f(r, X)=\Theta(r)$.
\end{abstract}

Keywords: Permutations; Sequences; Ramsey problems

\section{Introduction}

We consider the following Ramsey-type question. We say that two sequences $\left\{x_{i}\right\}_{i=1}^{t}$ and $\left\{y_{i}\right\}_{i=1}^{t}$ of distinct integers are similar if their entries are order-isomorphic, i.e., $x_{i}<x_{j}$ if and only if $y_{i}<y_{j}$ for all $1 \leqslant i<j \leqslant t$. For a given sequence $X$ and a positive integer $r$ a sequence $Y$ is Ramsey for $X$ if for every $r$-coloring of the entries of $Y$ there is a subsequence of $Y$ which is both monochromatic and similar to $X$. Denote by $f(r, X)$ the length of the shortest sequence $Y$ that is Ramsey for $X$, i.e.,

$$
f(r, X)=\min _{Y}|Y|
$$

where the minimum is taken over all Ramsey sequences for $X$. Moreover, let

$$
f(r, t)=\max _{X} f(r, X),
$$

*Supported in part by Simons Foundation Grant \#244712 and by a grant from the Faculty Research and Creative Activities Award (FRACAA), Western Michigan University. 
where the maximum is taken over all sequences $X$ with $|X|=t$.

Frankl, Rödl and the author [3] asked to determine for a fixed $t$ the order of magnitude of $f(r, t)$ as a function of $r$. Here we show that $f(r, t)=\Theta\left(r^{2}\right)$. Indeed, we give a stronger result identifying the asymptotic behavior of $f(r, X)$ for every $X$.

\section{Theorem 1.}

(i) Let $X$ be a monotone sequence, i.e., $X$ is similar to $(1,2, \ldots,|X|)$ or $(|X|, \ldots, 2,1)$. Then

$$
f(r, X)=\Theta(r)
$$

(ii) Let $X$ be a non-monotone sequence. Then

$$
f(r, X)=\Theta\left(r^{2}\right) .
$$

(The hidden constants depend only on $X$.)

It is also worth mentioning that the proof shows that for each $t$ there is a (universal) sequence $Y$ of length $O\left(r^{2}\right)$ which is Ramsey for every sequence $X$ of length $t$ and any number of colors $r$. Furthermore, the entries of such $Y$ colored by the majority color contain a subsequence similar to $X$.

\section{Proof of Theorem 1}

For (i) it is enough to observe that $(1,2, \ldots, r|X|)$ is Ramsey for $(1,2, \ldots,|X|)$, and similarly, $(r|X|, \ldots, 2,1)$ is Ramsey for $(|X|, \ldots, 2,1)$.

Now we prove (ii). First we show the lower bound. The proof is based on the ErdösSzekeres [4] theorem which says that any sequence $S$ of length $m$ contains a monotone subsequence of length $\lceil\sqrt{m}\rceil$. It is not difficult to observe (see, e.g., $[1,8]$ ) that the repetitive application of this result shows that $S$ can be partitioned into at most $\lfloor 2 \sqrt{m}\rfloor$ monotone subsequences. For the sake of completeness we prove a similar result here.

Let $X$ be any sequence of length $t$ which is non-monotone. Assume that $Y$ is Ramsey for $X$. We show that $|Y|>\left(\frac{r}{2}\right)^{2}$. Suppose not, i.e., $|Y| \leqslant\left(\frac{r}{2}\right)^{2}$. We will repeatedly

apply the Erdős-Szekeres theorem. We start with $Y$ of length $a_{0}=|Y| \leqslant\left(\frac{r}{2}\right)^{2}$ and find a monotone subsequence of length $\left\lceil\sqrt{a_{0}}\right\rceil$. Then we remove it from $Y$ obtaining a sequence of length $a_{1}=a_{0}-\left\lceil\sqrt{a_{0}}\right\rceil$ and repeat the whole process again. After the $i$-th step the length of the remaining sequence is given by the recursive formula

$$
a_{i+1}=a_{i}-\left\lceil\sqrt{a_{i}}\right\rceil .
$$

Let $N$ be the least integer for which $a_{N}=0$. We show that $N \leqslant r$. First observe that for each $i<N$, we have $a_{i} \geqslant 1$ and

$$
a_{i+1}=a_{i}-\left\lceil\sqrt{a_{i}}\right\rceil \leqslant a_{i}-\sqrt{a_{i}} \leqslant\left(\sqrt{a_{i}}-\frac{1}{2}\right)^{2}
$$


implying

$$
\sqrt{a_{i+1}} \leqslant \sqrt{a_{i}}-\frac{1}{2}
$$

and consequently,

$$
\sum_{i=0}^{N-1} \sqrt{a_{i+1}} \leqslant \sum_{i=0}^{N-1}\left(\sqrt{a_{i}}-\frac{1}{2}\right)
$$

Thus,

$$
\sqrt{a_{N}} \leqslant \sqrt{a_{0}}-\frac{N}{2} \leqslant \frac{r}{2}-\frac{N}{2}
$$

and after at most $r$ steps we end up with an empty sequence. Summarizing, we just found a decomposition of $Y$ into at most $r$ monotone subsequences. Now we color each monotone subsequence with a different color. Since $X$ is non-monotone, there is no monochromatic subsequence similar to $X$, a contradiction.

Next we show the upper bound. First we need some notation. Let $A$ and $P$ be $0-1$ matrices. We say that $A$ contains the $t \times t$ matrix $P=\left(p_{i, j}\right)$ if there exists a $t \times t$ submatrix $B=\left(b_{i, j}\right)$ of $A$ with $b_{i, j}=1$ whenever $p_{i, j}=1$. Otherwise we say that $A$ avoids $P$. Notice that we can delete rows and columns of $A$ to obtain the submatrix $B$ but we cannot permute the remaining rows and columns. Given a permutation $\pi$ of $t$ elements its permutation matrix is the $t \times t$ matrix $P_{\pi}=\left(p_{i, j}\right)$ whose entries are all 0 except that in column $i$, the entry $\pi(i)$ equals 1 , i.e., the only non-zero entries are $p_{\pi(i), i}$.

We will use the following result conjectured by Füredi and Hajnal [6] and proved by Marcus and Tardos [7]. Let $P$ be a permutation matrix. Denote by $g(P, m)$ the maximum number of ones in a 0-1 matrix of size $m \times m$ avoiding $P$. Then, due to Marcus and Tardos [7], there exists a positive constant $c=c(P)$ such that

$$
g(P, m) \leqslant c m .
$$

Let $X$ be a given sequence of $t$ different integers. (Here non-monotonicity is not required.) Without loss of generality we may assume that $X$ is a permutation of $\{1, \ldots, t\}$. Let $P_{X}$ be the corresponding permutation matrix and let $c=c\left(P_{X}\right)$ be as in (1) yielding

$$
g\left(P_{X}, m\right) \leqslant c m
$$

Set

$$
m=\lfloor c r\rfloor+1 .
$$

Now we define a sequence $Y$ which is a permutation of $\left\{1, \ldots, m^{2}\right\}$. Let

$$
\begin{aligned}
& Y=(1, \quad m+1, \quad 2 m+1, \quad 3 m+1, \quad \ldots, \quad(m-1) m+1, \\
& 2, \quad m+2, \quad 2 m+2, \quad 3 m+2, \quad \ldots, \quad(m-1) m+2, \\
& 3, \quad m+3, \quad 2 m+3, \quad 3 m+3, \quad \ldots, \quad(m-1) m+3, \\
& \begin{array}{lllll}
m, & \cdots & \cdots & \cdots & \cdots
\end{array}
\end{aligned}
$$

Clearly, $|Y|=\Theta\left(r^{2}\right)$. It remains to show that $Y$ is Ramsey for $X$. 
Let $A_{Y}$ be the following matrix of size $m \times m$ based on $Y$. The first $m$ elements of $Y$ form the first column in $A_{Y}$ in reverse order. The next $m$ elements of $Y$ form the second column in $A_{Y}$ in reverse order, etc. Thus,

$$
A_{Y}=\left(\begin{array}{rrrrr}
(m-1) m+1 & (m-1) m+2 & (m-1) m+3 & \ldots & m^{2} \\
(m-2) m+1 & (m-2) m+2 & (m-2) m+3 & \ldots & (m-1) m \\
(m-3) m+1 & (m-3) m+2 & (m-3) m+3 & \ldots & (m-2) m \\
\vdots & \vdots & \vdots & \vdots & \vdots \\
m+1 & m+2 & m+3 & \ldots & 2 m \\
1 & 2 & 3 & \ldots & m
\end{array}\right)
$$

Now let us arbitrarily color the elements of $Y$ with $r$ colors. We need to show that there is a monochromatic subsequence in $Y$ that is similar to $X$.

Clearly, every coloring of $Y$ uniquely induces a coloring of the entries of $A_{Y}$. Choose the most frequent color, say red, and let $A=\left(a_{i j}\right)$ be the 0-1 matrix of size $m \times m$ whose entries correspond to it. That means $a_{i j}=1$ if and only if the $i j$-entry in $A_{Y}$ is colored red. The key observation is the following: if $A$ does not avoid $P_{X}$, then $Y$ contains a monochromatic subsequence similar to $X$. By (3) and (2), we get that the number of ones in $A$ is at least

$$
\frac{m^{2}}{r}>c m \geqslant g\left(P_{X}, m\right)
$$

Hence, $A$ does not avoid $P_{X}$. This completes the proof of (ii).

\section{Concluding remarks}

It may be of some interest to study $f(r, t)$ in more detail. Theorem 1 implies that

$$
c_{1} r^{2} \leqslant f(r, t) \leqslant c_{2} r^{2}
$$

for some positive constants $c_{1}=c_{1}(t)$ and $c_{2}=c_{2}(t)$. For the sake of simplicity we did not attempt to optimize theses constants. The proof gives $c_{1}=\frac{1}{4}$ and this constant can be improved to $\frac{1}{2}$ by using a result of Brandstädt and Kratsch [2]. On the other hand, $c_{2}$ is entirely based on the result of Marcus and Tardos [7] and so is exponential in $t$ (see also a result of Fox [5]).

It would be also interesting to consider a similar question and study the growth of $f(r, t)$ for a fixed $r$ and large $t$.

For only two colors it is not difficult to see that

$$
f(2, t)=\Theta\left(t^{2}\right)
$$

Indeed, let $X=\left\{x_{i}\right\}_{i=1}^{t}$ be any sequence. Without loss of generality we may assume that $X$ is a permutation of $\{0, \ldots, t-1\}$. For the upper bound let us define $Y=$ $Y^{(1)} Y^{(2)} \ldots Y^{(t)}$, where $Y^{(i)}=\left(t x_{i}+x_{1}, t x_{i}+x_{2}, \ldots, t x_{i}+x_{t}\right)$ for $1 \leqslant i \leqslant t$. Now let us arbitrarily color the entries of $Y$ with two colors. Since each $Y^{(i)}$ is similar to $X$, we may 
assume that there is no monochromatic $Y^{(i)}$. Thus, there is a monochromatic subsequence $\left(y_{1}, y_{2}, \ldots, y_{t}\right)$ such that $y_{i} \in Y^{(i)}$ for $1 \leqslant i \leqslant t$. It is easy to see that such $\left(y_{1}, y_{2}, \ldots, y_{t}\right)$ is similar to $X$. Consequently, $Y$ is Ramsey for $X$ and $f(2, X) \leqslant|Y|=t^{2}$.

To see the lower bound of $(4)$ consider $X=\left(1,2, \ldots,\left\lfloor\frac{t}{2}\right\rfloor, t, t-1, \ldots,\left\lfloor\frac{t}{2}\right\rfloor+1\right)$. Let $Y$ be any Ramsey sequence for $X$. Clearly, $Y$ must contain many subsequences similar to $Z=\left(1,2, \ldots,\left\lfloor\frac{t}{2}\right\rfloor\right)$. Starting with $Y_{0}=Y$, we find a subsequence similar to $Z$ and remove it obtaining $Y_{1}$ (of length $|Y|-\left\lfloor\frac{t}{2}\right\rfloor$ ). We repeatedly continue the process of removing subsequences similar to $Z$ until we cannot longer find a subsequence similar to $Z$. Let $m$ denote the number of steps and $Y_{m}$ be the remaining sequence. Now we color $Y_{m}$ blue and $Y \backslash Y_{m}$ red. Since $Y_{m}$ contains no subsequence similar to $Z$, there is no blue subsequence similar to $X$ in $Y$. Therefore, there must be a red subsequence in $Y$ which is similar to $X$. In particular, there is a red subsequence similar to $X \backslash Z$. Since $Y \backslash Y_{m}$ is a disjoint union of $m$ (increasing) sequences similar to $Z$, each of these $m$ subsequences can contain at most one element of the (decreasing) sequence $X \backslash Z$. Thus, $m \geqslant t-\left\lfloor\frac{t}{2}\right\rfloor=\left\lceil\frac{t}{2}\right\rceil$ and so

$$
|Y| \geqslant\left|Y \backslash Y_{m}\right| \geqslant m\left\lfloor\frac{t}{2}\right\rfloor \geqslant\left\lceil\frac{t}{2}\right\rceil\left\lfloor\frac{t}{2}\right\rfloor \geqslant \frac{t^{2}-1}{4} .
$$

By recursively extending the above construction one can get an upper bound for any $r \geqslant 2$ and show that

$$
f(r, t) \leqslant t^{r}
$$

For example, for $r=3$ and a permutation $X=\left\{x_{i}\right\}_{i=1}^{t}$ of $\{0, \ldots, t-1\}$ it is enough to take $Y=Y^{(1)} Y^{(2)} \ldots Y^{(t)}$, where

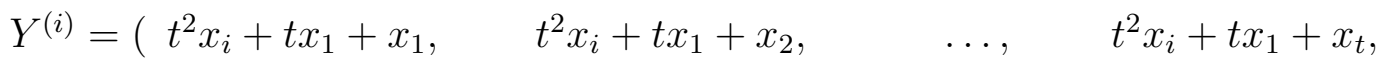

$$
\begin{aligned}
& t^{2} x_{i}+t x_{2}+x_{1}, \quad t^{2} x_{i}+t x_{2}+x_{2}, \quad \ldots, \quad t^{2} x_{i}+t x_{2}+x_{t},
\end{aligned}
$$

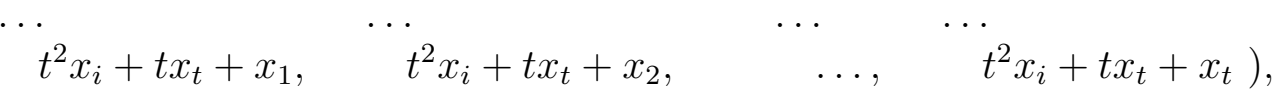

for $1 \leqslant i \leqslant t$. Observe that $Y$ is Ramsey for $X$. Hence, $f(3, X) \leqslant|Y|=t^{3}$.

The lower bound in (4) and the upper bound in (5) imply that for a fixed $r \geqslant 2$

$$
\Omega\left(t^{2}\right)=f(r, t)=O\left(t^{r}\right) .
$$

Determining the right order of magnitude of $f(r, t)$ as a function of $t$ remains open. ${ }^{1}$

\section{Acknowledgements}

The author would like to thank Boris Bukh for working on this problem. He was originally intended as a coauthor but later declined this. The author also would like to thank the referee for her/his valuable comments and suggestions, and in particular for pointing out reference [1].

\footnotetext{
${ }^{1}$ Very recently, together with Klimošová and Král' we showed that $f(r, t)=\Omega\left(\frac{t^{(r+1) / 2}}{\operatorname{polylog}(t)}\right)$. The proof uses some ideas from [5].
} 


\section{References}

[1] R. Bar-Yehuda and S. Fogel. Partitioning a sequence into few monotone subsequences. Acta Inform., 35(5):421-440, 1998.

[2] A. Brandstädt and D. Kratsch. On partitions of permutations into increasing and decreasing subsequences. Elektron. Informationsverarb. Kybernet., 22(5-6):263-273, 1986.

[3] A. Dudek, P. Frankl and V. Rödl. A note on universal and canonically coloured sequences. Combin. Probab. Comput., 18(5):683-689, 2009.

[4] P. Erdős and G. Szekeres. A combinatorial problem in geometry. Compos. Math., 2:463-470, 1935.

[5] J. Fox. Stanley-Wilf limits are typically exponential. arXiv:1310.8378.

[6] Z. Füredi and P. Hajnal. Davenport-Schinzel theory of matrices. Discrete Math., 103(3):233-251, 1992.

[7] A. Marcus and G. Tardos. Excluded permutation matrices and the Stanley-Wilf conjecture. J. Combin. Theory Ser. A, 107(1):153-160, 2004.

[8] J. Matoušek and E. Welzl. Good splitters for counting points in triangles. J. Algorithms, 13(2):307-319, 1992. 